\title{
Opto-chemische $\mathrm{NH}_{3}$-Sensoren im ppm und ppb-Bereich für den Einsatz in der Landwirtschaft
}

\author{
Norbert Winkler ${ }^{1}$, Sirko Pöhlmann ${ }^{1}$, Osgit Vogel ${ }^{1}$, Rainer Krage ${ }^{2}$, Martin Gorek ${ }^{2}$, Kristina \\ von Bobrutzki ${ }^{3}$, Werner Berg ${ }^{3}$ \\ ${ }^{1}$ Gesellschaft zur Förderung von Medizin-, Bio- und Umwelt-Technologien e.V., Fachsektion \\ Photonik und Sensorik, Felsbachstraße 7, 07745 Jena/Deutschland \\ ${ }^{2}$ GfG Gesellschaft für Gerätebau mbH, Klönnestraße 99, 44143 Dortmund/Deutschland \\ ${ }^{3}$ Leibniz-Institut für Agrartechnik Potsdam-Bornim e.V., Abteilung Technik in der Tierhal- \\ tung, Max-Eyth-Allee 100, 14469 Potsdam/Deutschland
}

\section{Zusammenfassung}

Für die Detektion von Ammoniak im ppm- und ppb-Bereich wurden empfindliche opto-chemische Schichtsysteme unter Verwendung der Sol-Gel-Technologie entwickelt und getestet. Mit diesen Schichten wurden opto-chemische Sensoren nach dem Prinzip eines Zweikanal-Photometers aufgebaut, mit denen ein reproduzierbarer Nachweis von Ammoniak im ppm-Bereich möglich ist. Die Sensoren besitzen keine Querempfindlichkeit $\mathrm{zu}_{2} \mathrm{~S}$ und sind für eine permanente Ammoniakdetektion geeignet. Dadurch ergeben sich sehr gute Möglichkeiten für den Einsatz dieser Systeme zur Überwachung von Stallluft. Unter Verwendung mehrerer Schichtsysteme ist auch ein Nachweis von Ammoniak im ppb-Bereich möglich. Es wurden umfangreiche Messungen mit den entwickelten Sensorsystemen in und um Stallanlagen durchgeführt und die Einsetzbarkeit der Sensoren zur Überwachung landwirtschaftlicher Anlagen nachgewiesen werden.

\section{Einleitung}

Der Überwachung der Ammoniakkonzentrationen in und in der Nähe von Stallanlagen kommt immer größere Bedeutung zu. So schreibt die Tierschutz-Nutztierhaltungsverordnung [1] zur Sicherung der Tiergesundheit maximale Obergrenzen von 10 ppm bzw. 20 ppm je nach Tierart vor. Zum Schutz der Ökosysteme empfiehlt die TA Luft (Fassung von 2002), dass die Gesamtbelastung von $10 \mu \mathrm{g} / \mathrm{m}^{3} \mathrm{NH}_{3}$ $(\approx 14 \mathrm{ppb})$ an keinem maßgeblichen Beurteilungspunkt überschritten wird. Allerdings wurden bei langfristigen Messungen teilweise erheblich höhere Konzentrationen in der Nähe von Stallanlagen detektiert. Für solche Messungen im ppb-Bereich stehen nur aufwendige Labormessverfahren zur Verfügung. Die gegenwärtig für Messungen im ppm-Bereich angebotenen Sensoren sind für die Überwachung der Stallluft nur bedingt einsetzbar [2]. So können die zu überwachenden Ammoniakkonzentrationen von 10 ppm nur mit einem großen Messfehler auf Grund der unteren Nachweisgrenze dieser Systeme detektiert werden. Außerdem besitzen sie z.B. deutliche Querempfindlichkeiten $\mathrm{zu}_{2} \mathrm{~S}$ oder sind für Dauermessungen (elektrochemische Sensoren) nicht geeignet.

Unter Verwendung einer $\mathrm{SiO}_{2}$-Matrix mit immobilisierten acidochromen Farbstoff, welche mit der Sol-Gel-Technologie auf einen Glasträger abgeschieden wird, wurden verschiedene ammoniaksensitive Schichten entwickelt und damit neuartige opto-chemische Sensoren in unterschiedlicher Bauform realisiert, die auch unter den Bedingungen der Stallluft einsetzbar sind. Die opto-chemischen Sensoren, welche nach dem Prinzip eines Zweistrahlphotometers arbeiten, messen die Absorptionsänderung der sensitiven Schichten in Abhängigkeit von der Ammoniakkonzentration.

\section{Schichtentwicklung}

Verschiedene ammoniaksensitive Schichten wurden für den opto-chemischen Nachweis entwickelt und systematisch getestet. Dazu wurden verschiedene acidochrome, $\mathrm{pH}$-sensitive Indikatorfarbstoffe 
(Triphenylmethan-Farbstoffe) physikalisch in eine saure $\mathrm{SiO}_{2}$-Gel-Matrix immobilisiert. Mit Hilfe des Sol-Gel-Verfahrens [3] wurden ca. $250 \mathrm{~nm}$ dicke Schichten beidseitig auf Glasträgern abgeschieden und auf ihre Ammoniaksensitivität getestet. Die besten Ergebnisse wurden mit dem System BP135 erzielt. Dabei wurde der acidochrome Indikator in ein sauer hydrolisiertes $\mathrm{SiO}_{2}$-Sol (4,2 \% Feststoffgehalt, $70 \%$ Ethanol) immobilisiert.

Bild 1 zeigt die Transmissionsverläufe des entwickelten Schichtsystems bei unterschiedlichen Ammoniakkonzentrationen. Die Absorptionsbande des Schichtsystems BP135 befindet sich bei $592 \mathrm{~nm}$ und kann zur Detektion von Ammoniak genutzt werden. Die Änderung der Extinktion und die damit erreichbare Auflösung für die zu detektierenden Ammoniakkonzentrationen sind im Konzentrationsbereich bis 150 ppm ausreichend groß. Bild 2 zeigt die Änderung der Transmission des Schichtsystems bei einer Wellenlänge von $592 \mathrm{~nm}$ für kleine Ammoniakkonzentrationen. Die Änderungen sind für den sicheren Nachweis von $1 \mathrm{ppm}$ Ammoniak ausreichend. Die $\mathrm{t}_{90}$-Zeiten betragen $200 \mathrm{~s}$ bei einer Ammoniakkonzentration von $1 \mathrm{ppm}$ und ca. $300 \mathrm{sec}$ bei $2 \mathrm{ppm}$.

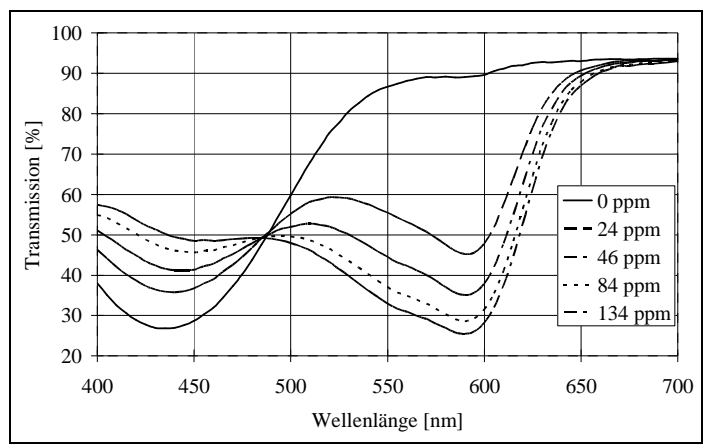

Bild 1 Änderung der Transmission für das Schichtsystem BP 135 in Abhängigkeit von der Ammoniakkonzentration

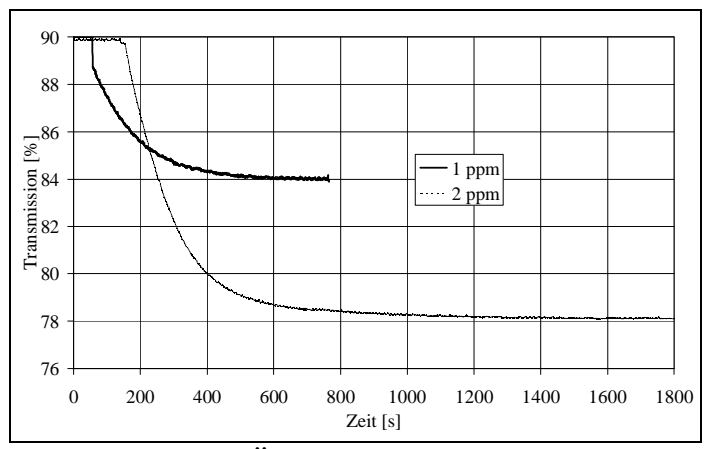

Bild 2 Zeitliche Änderung der Transmission des Schichtsystems BP135 bei Ammoniakkonzentrationen von $1 \mathrm{ppm}$ und $2 \mathrm{ppm}$

\section{Sensorsysteme und Tests}

Das opto-chemische Sensorsystem AS5 wurde nach dem Prinzip eines Zweistrahl-Photometers aufgebaut und mit den entwickelten sensitiven Schichten ausgestattet. Bild 3 gibt einen Überblick über den realisierten Gesamtaufbau. Für das Schichtsystem BP135 kam eine LED mit der Wellenlänge $590 \mathrm{~nm}$ zum Einsatz. Die Messung der Sensorsignale erfolgt als 2-Kanal-Messung mit Referenz- und Messkanal, Dunkelstromsubtraktion und Basiskorrektur. Die Messkammer wurde so aufgebaut, dass sie bis zu sieben sensitive Schichtsysteme aufnehmen kann. Dadurch kann das Sensorsystem so modifiziert werden, dass sowohl Ammoniakkonzentrationen im ppm- als auch im ppb-Bereich detektiert werden können. Der Nachweis im ppm-Bereich erfolgt mit einem sensitiven Schichtsystem. Für die Detektion der Ammoniakkonzentration im ppb-Bereich wurden fünf sensitive Schichtsysteme eingesetzt. Dadurch werden geringste Änderungen in der Schichttransmission verstärkt und für das Sensorsystem messbar.

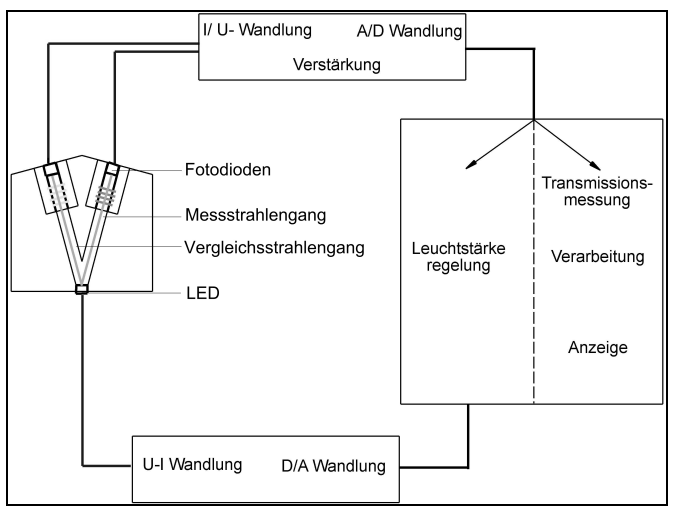

Bild 3 Prinzipskizze des Sensorsystems AS5 für die Ammoniakdetektion mit variabler Anzahl sensitiver Schichten 
In den Bildern 4 und 5 werden für die unterschiedlichen Systeme die Kalibrierkurven dargestellt. Es ist zu erkennen, dass durch die Variation der Anzahl der sensitiven Schichten eine Anpassung des Sensorsystems an den ppm- bzw. ppb-Bereich möglich ist. Die unteren Nachweisgrenzen betragen dabei $0,5 \mathrm{ppm}$ bei einem sensitiven Schichtsystem und $20 \mathrm{ppb}$ bei fünf Schichtsystemen.

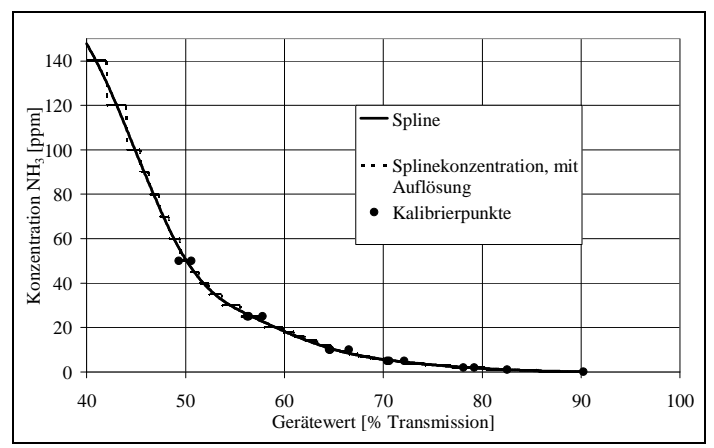

Bild 4 Kalibrierkurve des Ammoniaksensors AS5 mit einem sensitiven Schichtsystem für die Ammoniakdetektion im ppm-Bereich $\left(25^{\circ} \mathrm{C}, 50 \%\right.$ r.F. $)$

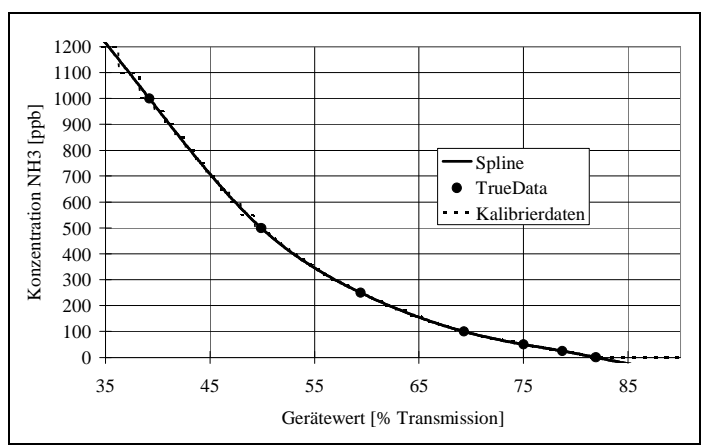

Bild 5 Kalibrierkurve des Ammoniaksensors AS5 mit fünf sensitiven Schichtsystemen für die Ammoniakdetektion im ppb-Bereich $\left(25^{\circ} \mathrm{C}, 50 \%\right.$ r.F. $)$

Systematische Untersuchungen mit den aufgebauten Sensoren zeigten eine sehr gute Nullpunktstabilität und Reproduzierbarkeit der Messwerte auf. In Bild 6 ist das Ergebnis einer Langzeituntersuchung über 28 Tage dargestellt. Dabei wurde das Sensorsystem permanent einer Ammoniakkonzentration von $10 \mathrm{ppm}$ ausgesetzt. Querempfindlichkeiten $\mathrm{zu} \mathrm{H}_{2} \mathrm{~S}$, welches in Stallanlagen häufig auftritt, konnten nicht detektiert werden (Bild 7). Auf Grund dieser Eigenschaften ist eine gute Einsetzbarkeit des Sensorsystems in Stallanlagen gegeben.

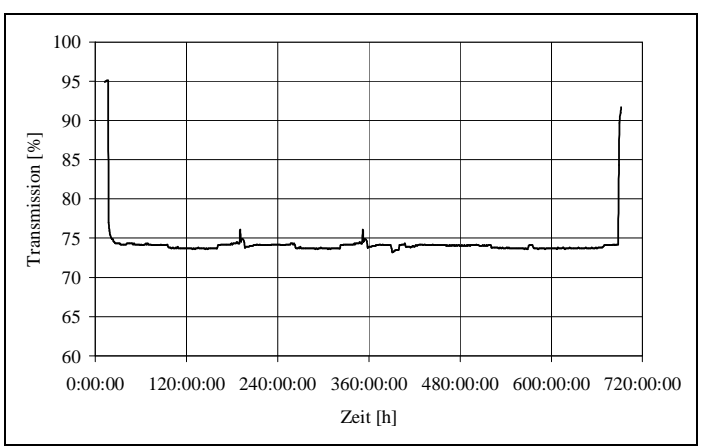

Bild 6 Sensortransmission bei einer Dauermessung mit dem Sensor AS 5 (ein sensitives Schichtsystem) bei einer Ammoniakkonzentration von 10 ppm

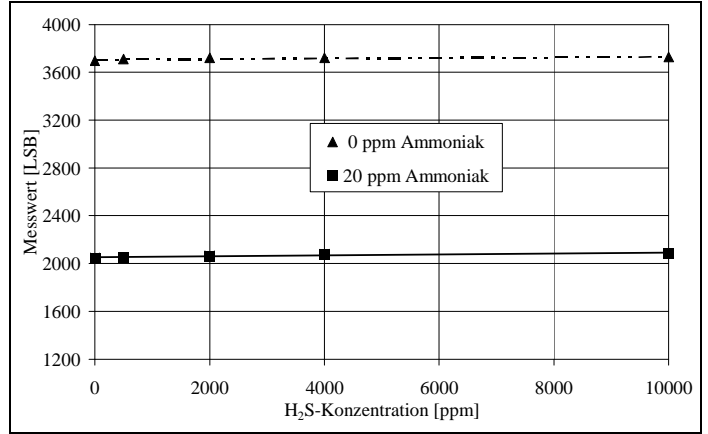

Bild 7 Sensorsignal in Abhängigkeit von der $\mathrm{H}_{2} \mathrm{~S}$ Konzentration für Gasströme mit 0 ppm bzw. 20 ppm Ammoniak

\section{Tests in der Landwirtschaft}

Basierend auf dem in Bild 3 dargestellten Messprinzip und den Ergebnissen der durchgeführten Tests wurde ein mit kommerziellen Systemen kompatibler Sensoraufbau für Messungen im ppm-Bereich realisiert (Bild 8) und dieser in umfangreichen Untersuchungen in landwirtschaftlichen Einrichtungen (Hähnchenmast, Schweine- und Rinderstall) eingesetzt. In Bild 9 sind die Messergebnisse der getesteten Sensoren aus einem Stall zur Hähnchenmast über einen Mastzyklus dargestellt. Zum Vergleich wurden gleichzeitig Messungen mit elektro-chemischen Ammoniaksensoren durchgeführt. Bild 9 zeigt, dass ab ca. 25 Tagen die Messergebnisse deutlich voneinander abweichen und die elektrochemischen Sensoren kleinere Messwerte liefern. In einem nach dem Stalleinsatz durchgeführten Empfindlichkeitstest konnte gezeigt werden, dass die elektro-chemischen Sensoren eine deutliche Empfindlichkeitsverringerung aufweisen (Bild10). Die Abnahme der Empfindlichkeit korreliert mit der Verringerung der Messsignale aus den Stallmessungen. Analoge Ergebnisse wurden bei den Messungen im Schweinestall erzielt.

Bild 11 zeigt die Ergebnisse einer Ammoniakmessung außerhalb eines Rinderstalls. Dazu wurde ein Ammoniaksensor AS5 für den ppb-Bereich mit fünf sensitiven Schichtsystemen entsprechend Bild 3 
eingesetzt. Vergleichsmessungen erfolgten mit einem Multigas-Monitor (INNOVA 1312, Bruehl \& Kjaer). Die Ergebnisse zeigen unter Beachtung der auftretenden Messfehler eine gute Übereinstimmung. Geringfügige Unterschiede können auch durch geringe Abweichungen der Gasentnahmestellen hervorgerufen sein.
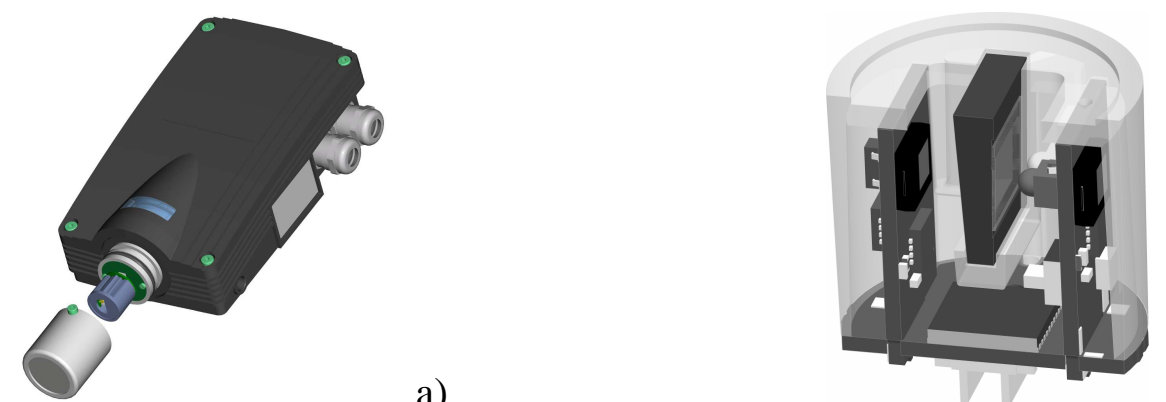

a)

b)

Bild 8 Mit kommerziellen Systemen kompatibler Sensoraufbau; a) Sensor für stationäre Messungen b) Sensoraufbau mit sensitiver Schicht (Bildmitte), LED und Photodioden

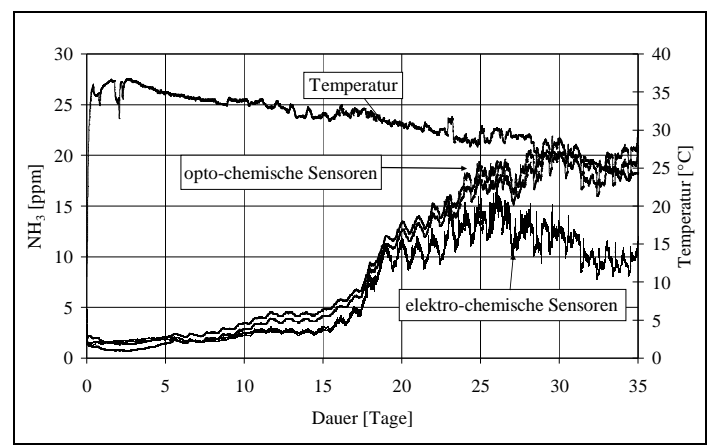

Bild 9 Ammoniakmessung in einem Stall zur Hähnchenmast mit opto- und elektro-chemischen Sensoren

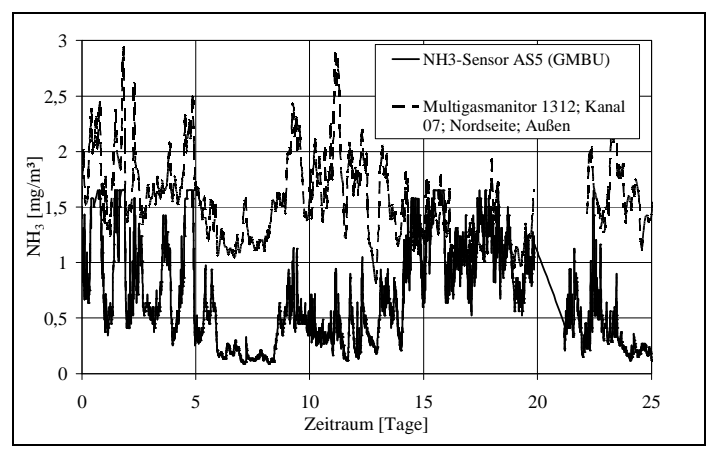

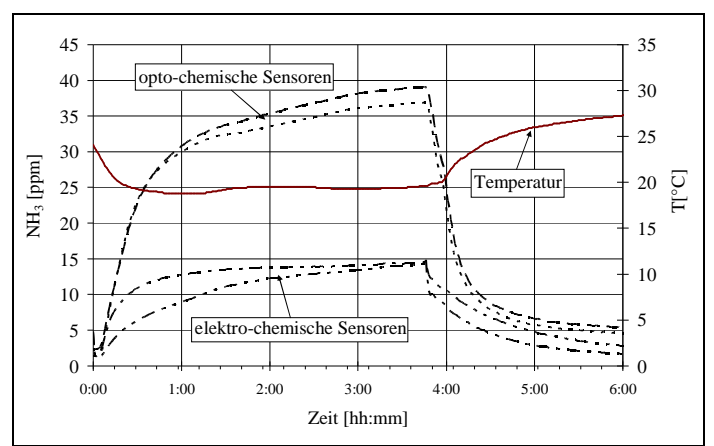

Bild 10 Empfindlichkeitstest der im Stall (Bild 9) eingesetzten Sensoren (35 ppm Ammoniak)

Bild 11 Ammoniakkonzentration außerhalb einer Rindermastanlage; gemessen mit dem Ammoniaksensor AS5 (fünf sensitive Schichtsysteme) und einem Multigas-Monitor INNOVA 1312 (1 ppm Ammoniak entspricht $0,7 \mathrm{mg} / \mathrm{m}^{3}$ )

\section{Danksagung}

Diese Arbeit wurde vom Bundesministerium für Wirtschaft und Technologie über die Arbeitsgemeinschaft industrielle Forschung (ZIM-KF 2108604WM8) gefördert. Wir danken für die wertvolle Unterstützung.

\section{Literatur}

[1] TierSchNutztV- Tierschutz-NutztierhaltungsverordnungVerordnung zum Schutz landwirtschaftlicher Nutztiere und anderer zur Erzeugung tierischer Produkte gehaltener Tiere bei ihrer Haltung. 22. August 2006 (BGBl. I Nr. 41 vom 31.08.2006 S. 2043)

[2] W. Grotz, B. Rathemer, A. Gronauer und H. Schön: „Schadgas geregelte Lüftungstechnik für Mastschweine"; Agrartechnische Forschung 7 (2001) Heft 2, S. 57

[3] C.J. Brinker, G. Scherer: „Sol-Gel-Science, The Physics and Chemistry of Sol-Gel Process“. Academic Press London 1990 